\title{
The potential application of personalized medicine to antipsychotic treatment: genetic prediction of extrapyramidal and metabolic adverse effects
} Bernard Lerer

\author{
Address: Biological Psychiatry Laboratory, Department of Psychiatry, Hadassah - Hebrew University Medical Center, Jerusalem, Israel \\ from International Society on Brain and Behaviour: 3rd International Congress on Brain and Behaviour \\ Thessaloniki, Greece. 28 November - 2 December 2007 \\ Published: 17 April 2008 \\ Annals of General Psychiatry 2008, 7(SuppI I):S34 doi:10.II86/I744-859X-7-SI-S34
}

This abstract is available from: http://www.annals-general-psychiatry.com/content/7/SI/S34

(c) 2008 Lerer; licensee BioMed Central Ltd.

Extrapyramidal symptoms (EPS) such as antipsychoticinduced parkinsonism (AIP) and tardive dyskinesia (TD) are the most prominent adverse effects of first generation (or typical) antipsychotics (FGAs). Most clinicians would agree that concerns regarding EPS are the most important factor limiting prescription of FGAs. As a consequence, and not because of greater efficacy, second generation (or atypical) antipsychotics (SGAs) are rapidly becoming the first line treatment for schizophrenia and other psychotic states. The extensive use of SGAs has uncovered serious adverse effects of these agents. Prominent among these are weight gain, which may be extensive and widespread, hyperlipidemia and diabetes. Although EPS are common among patients administered FGAs and metabolic syndrome is frequent in patients who receive SGAs, a substantial proportion do not develop these adverse effects. Genetic factors may contribute substantially to this variable, inter-individual susceptibility. Identification of the specific genes involved could open the way to the application of a personalized medicine approach to the prescription of antipsychotic drugs. The most likely model is multifactorial and polygenic and several different genes are likely to be involved. Extensive research is under way to identify these genes and develop valid and clinically applicable predictive models. Associations with meta-analytic support include the role of variants in the dopamine D3 receptor gene (DRD3) and the 5-HT2A receptor gene (HTR2A) in susceptibility to TD. More recent findings from of our group include the potential role of the regulator of G protein signaling 2 gene (RGS2) in susceptibility to AIP. In parallel, a search is under way for genes that confer susceptibility to weight gain and metabolic syndrome in patient treated with SGAs. Our group and others have focused on genes that influence lipid metabolism.
The results of whole genome analyses can be expected to further advance the field. Considering that genetic factors may also influence therapeutic effects, it is clear that personalized medicine in the field of antipsychotic treatment will not involve a single genetic test but a multivariate tapestry of genetic and environmental factors that will need to be taken into consideration in the context of a predictive algorithm. Development of this algorithm is less challenging at the technological and computational level than the implementation of urgently-needed, appropriately designed clinical trials to identify the genes involved. 